\title{
Longitudinal associations between specific symptoms of depression : Network analysis in a prospective cohort study
}

\section{Savelieva, Kateryna}

2021-01-01

Savelieva , K , Komulainen , K, Elovainio , M \& Jokela , M 2021 , ' Longitudinal associations between specific symptoms of depression : Network analysis in a prospective cohort study ', Journal of Affective Disorders , vol. 278 , pp. 99-106 . https://doi.org/10.1016/j.jad.2020.09.024

http://hdl.handle.net/10138/334440

https://doi.org/10.1016/j.jad.2020.09.024

cc_by_nc_nd

acceptedVersion

Downloaded from Helda, University of Helsinki institutional repository.

This is an electronic reprint of the original article.

This reprint may differ from the original in pagination and typographic detail.

Please cite the original version. 
This is an Accepted Manuscript of an article published by Elsevier in Journal of Affective Disorders. The final publication is available via https://doi.org/10.1016/j.jad.2020.09.024

\section{Kateryna Savelieva $^{1}$, Kaisla Komulainen ${ }^{1}$, Marko Elovainio ${ }^{2}$, Markus Jokela ${ }^{1}$ (2021)} Longitudinal associations between specific symptoms of depression: Network analysis in a prospective cohort study, Journal of Affective Disorders, 278: 99-106.

\footnotetext{
${ }^{1}$ Department of Psychology and Logopedics, Faculty of Medicine, University of Helsinki, Finland.

${ }^{2}$ Research Program Unit, Faculty of Medicine, University of Helsinki and Finnish Institute for Health and Welfare, Helsinki, Finland.
}

Correspondence concerning this article should be addressed to Dr. Kateryna Savelieva, Department of Psychology and Logopedics, Faculty of Medicine, P.O. Box 21 (Haartmaninkatu 3), 00014 University of Helsinki, Helsinki, Finland. E-mail: kateryna.savelieva@helsinki.fi Funding: This work was supported by the Academy of Finland (grant number 311578 for MJ and 329224 for ME). 


\section{Highlights}

- Changes in depressive symptoms were analyzed using network analysis.

- Improvements in symptoms were largely related to subsequent improvements of other symptoms.

- Deterioration in symptoms was associated with other symptoms' worsening over time.

- $\mathrm{Sad} / \mathrm{depressed}$ mood, diminished interest, suicidal ideation were the most central symptoms.

- Symptom networks did not differ between those taking vs. not taking psychotropic medication. 


\begin{abstract}
Background. Network perspective to mental disorders suggests that depression develops due to interrelated associations between individual symptoms rather than due to a common cause. However, it is unclear whether long-term longitudinal associations between specific symptoms of depression demonstrate coherent patterns. We examined the temporal sequences and changes in depressive symptoms over time, and whether some symptoms are more central than others in inducing changes in the rest of the symptoms over time. We also compared the network structure of depressive symptoms between people who were and were not taking medication for depression or anxiety.
\end{abstract}

Methods. Data were from the Survey of Health, Aging and Retirement in Europe, with five follow-ups conducted between 2004 and 2017. Participants who had data on depressive symptoms from at least two study waves were analyzed $(n=72,971)$. Depressive symptoms were self-reported using the 12-item EURO-D scale.

Results. All individual symptoms were longitudinally associated with each other. Changes in sad or depressed mood, diminished interest, and suicidal ideation were the most strongly associated with changes in other symptoms. There were no consistent differences in symptom associations between individuals taking versus not taking psychotropic medication.

Limitations. Depressive symptoms were self-reported and measured every two years, which may dilute some short-term temporal sequences of the symptoms.

Conclusions. Our findings demonstrate differences between depressive symptoms in their long-term associations with other depressive symptoms in the general population. Changes in sad or depressed mood, diminished interest, and suicidal ideation have the strongest associations with changes in the rest of the symptoms.

Keywords: depressive symptoms, network analyses, longitudinal data, symptom-level approach, cohort study 


\section{Introduction}

Depression includes cognitive, emotional, and somatic symptoms that can form multiple symptom combinations in the diagnosis of clinical depression (Fried, 2017; Fried and Nesse, 2015). Recent research has challenged the idea that these depressive symptoms are representations of an underlying disease of depression ("common cause"). Instead, depression is suggested to develop as a result of causal interactions between symptoms rather than as a result of underlying common cause (Borsboom, 2017; Cramer et al., 2010). A growing number of studies have applied a network approach to assess the structure of psychopathology and the patterns between specific symptoms of depression (for a review, see Fried et al., 2017). The majority of previous studies have been cross-sectional but some studies have examined longitudinal networks of depressive symptoms (Bos et al., 2017; Bringmann et al., 2015; Groen et al., 2019) or affective states more generally (Klippel et al., 2018; Snippe et al., 2017).

Some symptoms may be more influential than others in triggering the onset of further symptoms (Borsboom, 2017). In general, all symptoms of depression tend to change in unison over time, that is, improvements and deteriorations in symptoms are correlated across individual symptoms (Bringmann et al., 2015; Groen et al., 2019). Some studies have reported that an increase in suicidal thoughts and loss of pleasure may be particularly strongly related to increase in other symptoms over short time periods in weekly assessments (Bos et al., 2017; Bringmann et al., 2015). Previous studies have mainly focused on how the presence of a specific symptom at one timepoint is related to the presence of other symptoms at a subsequent timepoint. Less is known how a change in a specific symptom is related to changes in all other symptoms. Moreover, most of the longitudinal studies have focused on short-term assessments of depressive symptoms across days or weeks, and it is unknown 
whether the symptom-specific associations can be observed over longer time intervals of several years.

The symptom associations of depression may also depend on other health-related factors. Use of antidepressant medication is relatively common in the general population, with on average $7.2 \%$ of Europeans using antidepressant medication (Lewer et al., 2015). Antidepressant medication may influence specific depressive symptoms differently (Hieronymus et al., 2016; Lisinski et al., 2020). However, it is not known whether antidepressant medication also influences the associations between depressive symptoms over time. Given that antidepressant medication is targeted to reduce symptoms of depression, one could hypothesize that antidepressant medication strengthens the positive effects of improvements between depressive symptoms and attenuates the negative effects of deterioration between depressive symptoms.

The present study examined the long-term networks of depressive symptoms in a large panel study that covers most European countries. The first aim was to examine the longitudinal associations of depressive symptoms and changes (improvement or worsening) of depressive symptoms over time. The second aim was to examine whether some symptoms are more central than others in triggering other symptoms over the long-term course of depression. Based on previous studies (Bos et al., 2017; Bringmann et al., 2015), we expect that at least suicidal ideation and loss of pleasure/interest will be important symptoms in triggering changes in other symptoms. Finally, the third aim was to compare the long-term dynamic networks of depressive symptoms between people who used and did not use medication for depression and anxiety. We hypothesized that taking medication would amplify the positive associations of improvements between symptoms and attenuate the negative associations of deteriorations between specific symptoms over time. 


\section{Methods}

\section{Sample}

The Survey of Health, Ageing and Retirement in Europe (SHARE) is a cross-national panel database covering 27 European countries and Israel. It contains data on health, socioeconomic status, and social and family networks of about 140,000 persons aged 50 or older and their spouses. To date, SHARE has collected six panel waves (Wave 1, 2, 4, 5, 6, and 7), as well as data on retrospective life histories (SHARELIFE, Wave 3). For methodological details on SHARE, see Börsch-Supan et al. (2013); the response or retention rates are reported elsewhere (Bergmann et al., 2019).

In this study, we used all available data on depressive symptoms from SHARE panel waves, which comprised five follow-ups: from wave 1 (taking place in 2004-2006) to 2 (2006-2010), 2 to 4 (2010-2012), 4 to 5 (2013), 5 to 6 (2015), and 6 to 7 (2017) (BörschSupan, 2019a, 2019b, 2019c, 2019d, 2019e, 2019f). A total of 117,278 participants $(263,748$ total person-observations) aged 20-100 years had data on all depressive symptoms from any of the study waves. Of those, 72,971 participants (146,470 total person-observations) had data from at least two follow-ups and comprised the analytical sample for this study. The results from the attrition analysis show that the participants with a higher number of depressive symptoms were more likely to drop out during the follow-ups ( $\mathrm{OR}=1.02,95 \%$ $1.01,1.02)$. The associations between the specific depressive symptoms and the loss to follow-up are shown in Supplementary Table 1.

SHARE was reviewed and approved by the Ethics Committee of the University of Mannheim (Waves 1-4) and by the Ethics Council of the Max Planck Society (Waves 5-7). In addition, the country implementations of SHARE were reviewed and approved by the respective ethics committees or institutional review boards whenever this was required.

\section{Measures}


Depressive symptoms. Depressive symptoms were self-reported using the 12 -item EURO-D scale (Prince et al., 1999b, 1999a). The scale was originally developed to harmonise data on late-life depression from population-based studies across European countries (Prince et al., 1999b). The EURO-D items were originally derived from the Geriatric Mental State scale and comprise sad or depressed mood, pessimism, suicidal ideation, guilt, sleep, loss of interest, irritability, appetite, fatigue, concentration, enjoyment, and tearfulness. Individual symptoms were scored as 0 ('not present') and 1 ('present') (Prince et al., 1999b). Based on the initial validation in the cross-European study on depression prevalence (EURODEP), the scale has an adequate internal consistency and validity (Prince et al., 1999b, 1999a). Further studies using SHARE data support the crosscultural validity of the scale (Castro-Costa et al., 2008).

Medication for depression or anxiety. All survey respondents were asked whether they were currently taking drugs at least once a week for depression or anxiety. Respondents who reported taking medication for depression or anxiety were coded as 1 ('treatment group'), and those who did not report taking medication for depression or anxiety - as 0 ('no treatment' group).

Control variables. We adjusted all analyses for age, sex, mean level of depression in the country of interview, and follow-up time.

\section{Statistical analysis}

Modelling longitudinal network systems. First, we examined time-lagged associations between depressive symptoms by conducting a model where a depressive symptom at each time point $(t)$ was used as a predictor of that same symptom at the subsequent time point $(t+1$; to control for possible autoregressive effects) and of all other symptoms at the subsequent time point $(t+1)$. For this, separate logistic regression analyses were performed for each symptom using the Stata logit command. We conducted all the 
analyses using the robust cluster estimator to account for the dependence of measurements within the same individual. The analyses were adjusted for age, sex, mean level of depression in the country of interview, and follow-up time.

We then saved the estimates from logistic regression analyses and used these estimates as the edge weights to visualize the networks of symptoms using the qgraph package in R. In the networks, each node represents a presence of a symptom and each edge is the association of all symptoms at one time point with a symptom of interest at a subsequent time point. The autoregressive associations were not shown in the networks and also not included in the calculation of the centrality indices, since we were not interested in showing the time-lagged associations within the same symptoms. Two centrality indices out-strength and in-strength - were used to examine symptoms' centralities in the networks. Out-strength (i.e., sum of outgoing edge weights from a specific node) indicates how connected a symptom was at one time point to other symptoms at a subsequent time point; in-strength (i.e., sum of ingoing edge weights to a specific node) reflects how likely a symptom was to follow after other symptoms being present at a prior time point. Centrality indices were visualized using 'centralityPlot' command in the qgraph package in R.

Modelling of change in networks. We further tested whether changes in all depressive symptoms within each follow-up (e.g., from time 1 to time 2) were related to the presence of each depressive symptom at the end of the follow-up (e.g., time 2) when that symptom was present at baseline (e.g., time 1). A change score was created for each symptom by subtracting the symptom score at time-point $t$ from the same symptom score at time-point $t+1$. We categorized the change score into (a) a score characterizing improvement of symptoms $(0=$ remaining the same or worsening, $1=$ improving $)$ and (b) a score characterizing worsening of symptoms $(0=$ remaining the same or improving, $1=$ worsening). We performed separate logistic regression analyses with robust cluster estimator 
for each depressive symptom at $t+1$ as the outcome and (a) improvement or (b) worsening of all other symptoms from $t$ to $t+1$ as the predictors when the symptom used as the outcome was present at time $t$. Improvement and worsening of symptoms were analyzed in separate models. The analyses were adjusted for age, sex, mean level of depression in the country of interview, and follow-up time. Likewise, we used the estimates from the logistic regression analyses as the edge weights to visualize the networks of depressive symptoms using qgraph package, and plot the centrality indices (in-strength and out-strength) using 'centralityPlot' command in the qgraph package in $\mathrm{R}$.

\section{Differences in networks between 'treatment' and 'no treatment' groups. We} repeated the change models in two groups: (a) people who reported taking medication for depression or anxiety ('treatment' group) and (b) those who did not report taking medication for depression and anxiety ('no treatment' group). To ensure comparability between the 'no treatment' group and 'treatment' group, we selected only those respondents in the 'no treatment' group who have three or more depressive symptoms at the baseline of each follow-up, so that the mean level of depressive symptoms became similar in both groups ( $M$ $=4.4$ in 'treatment' group and $M=4.5$ in 'no treatment' group). The 'treatment' group comprised 6,365 participants and the 'no treatment' group included 33,490 participants. We used the centrality indices (in-strength and out-strength) to compare the networks of depressive symptoms in these two groups. Confidence intervals for centrality indices were calculated by resampling values for the centrality indices 500 times from a distribution which was defined by the edge weight (distribution mean) and standard error (distribution standard deviation) obtained from the regression analysis.

All statistical analyses were performed in Stata 15 (StataCorp, 2017) and RStudio version 1.1.463 (RStudio Team, 2015). 


\section{Results}

\section{Descriptive statistics}

The mean age of participants at baseline was $63.2(\mathrm{SD}=9.93), 57 \%$ were female. The prevalence of depressive symptoms in the whole sample is shown in Table 1 . The most prevalent symptoms were being sad or depressed over the last month (38.8\%), fatigue (34\%), trouble with sleep (33.8\%), and irritability (27.6\%).

Table 1. Frequency of depressive symptoms in the analytical sample ( $n=146,470$ total person-observations).

\begin{tabular}{|c|c|}
\hline EURO-D item & Frequency \\
\hline Sad or depressed last month & $38.8 \%$ \\
\hline Hopes for the future ${ }^{a}$ & $15.5 \%$ \\
\hline Felt would rather be dead & $6.6 \%$ \\
\hline Feels guilty & $8.3 \%$ \\
\hline Trouble sleeping & $33.8 \%$ \\
\hline Less interest in things & $8.2 \%$ \\
\hline Irritability & $27.6 \%$ \\
\hline Appetite ${ }^{b}$ & $7.7 \%$ \\
\hline Fatigue & $34.0 \%$ \\
\hline Concentration $^{c}$ & $17.2 \%$ \\
\hline Enjoyment $^{\mathrm{d}}$ & $11.9 \%$ \\
\hline Tearfulness & $24.6 \%$ \\
\hline
\end{tabular}

\footnotetext{
${ }^{\mathrm{a}}$ No hopes mentioned

${ }^{\mathrm{b}}$ Diminution in desire for food

${ }^{c}$ Difficulty in concentrating on entertainment or reading

${ }^{\mathrm{d}}$ Fails to mention any enjoyable activity
} 


\section{Time-lag model}

Figure 1.A shows the dynamic network of depressive symptoms in the total sample based on the time-lag model. All associations in the network were positive indicating that presence of a symptom at one time point was related to presence (but not absence) of other depressive symptoms at subsequent time point. Figure 1.B shows the centrality indices for the network in the total sample. Suicidal ideation ("Felt would rather be dead") had the highest out-strength estimate, suggesting that suicidal ideation was most connected to other symptoms in the network over time. Suicidal ideation, along with diminished interest and being sad or depressed, also had the highest in-strength estimate, indicating that these symptoms were likely to follow after the presence of other symptoms.

\section{Change model}

Figure 2.A presents the dynamic network of improvement in depressive symptoms over time in the total sample based on the change model. Most of the associations were negative (shown in red color) indicating that improvement in symptoms over the follow-up was associated with subsequent absence of a symptom at the end of the follow-up when that symptom of interest was present at the beginning of the follow-up. Sad or depressed mood had the highest out-strength estimate, suggesting that improvement in sad or depressed mood over the follow-up was most connected with subsequent absence of other symptoms at the end of the follow-up (Figure 2.B). Suicidal ideation had the highest in-strength estimate, suggesting that improvement in suicidal ideation was particularly likely to occur after improvement in other symptoms (Figure 2.B).

Figure 3.A presents the dynamic network of deterioration of depressive symptoms over time in the total sample based on the change model. All associations were positive (in blue color) indicating that deterioration of symptoms over the follow-up was associated with subsequent deterioration of other symptoms at the end of the follow-up. Suicidal ideation and 
less interest in things had the highest out-strength estimate, suggesting that deterioration in these two symptoms over the follow-up was most connected with deterioration in all other symptoms at the end of the follow-up (Figure 3.B). Sad or depressed mood had the highest in-strength estimate, suggesting that deterioration in sad or depressed mood was particularly likely to occur after deterioration of all other symptoms (Figure 3.B).

\section{'Treatment' and 'no treatment' group}

Supplementary Table 2 shows the odds ratios for the associations of medication use for depression or anxiety and having depressive symptoms. In the cross-sectional analyses, medication use was associated with lower probability of some depressive symptoms (e.g., sad or depressed mood, irritability, and pessimism) and higher probability of other symptoms (e.g., suicidal ideation, feeling guilty, and diminished interest in things). In the longitudinal analyses, medication use was related to higher probability of all depressive symptoms.

The dynamic networks of improvement and deterioration of depressive symptoms over time were similar between 'treatment' and 'no treatment' groups (Supplementary Figure 1-2). The centrality indices were also largely similar between the two groups (Figure 4). In the change model for improvement of depressive symptoms, sad or depressed mood had the highest out-strength estimates in both groups, without any difference between the 'treatment' and 'no treatment' groups (Supplementary Table 3). However, participants in 'no treatment' group had higher out-strength estimates for tearfulness and irritability, but lower for suicidal ideation, as compared to 'treatment' group (Supplementary Table 3). Also, suicidal ideation and diminished interest had the highest in-strength estimates for both groups, whereas sad or depressed mood had higher in-strength and pessimism had lower in-strength in 'no treatment' group (Supplementary Table 3). In the change model for deterioration of depressive symptoms, suicidal ideation and less interest in things had the highest out-strength estimates in both groups, without any difference between the 'treatment' and 'no treatment' groups 
(Supplementary Table 4). However, sad or depressed mood had the higher in-strength estimate in 'treatment group'; whereas less interest in things had higher in-strength estimate in 'no treatment group' (Supplementary Table 4).

\section{Discussion}

This study examined longitudinal network associations between depressive symptoms in the general population using data from the prospective cohort study covering most European countries. We found that all symptoms were directly or indirectly connected with each other and that a presence of a symptom at one time point was related to higher probability of other depressive symptoms later in time. Improvement in symptoms over time was associated with subsequent improvement in most other symptoms, whereas worsening of symptoms over time was related to deterioration of other symptoms over time.

We found some support for the hypothesis that some symptoms are more important than others in the longitudinal dynamics of symptoms. The two core symptoms for depression diagnosis - sad or depressed mood and diminished interest in things - along with suicidal ideation were the most central symptoms in the network of changes in depressive symptoms over time. Our findings are consistent with studies showing that depressed mood and anhedonia are most reactive to changes in other symptoms (i.e., high in-strength estimate), whereas suicidal ideation may induce other symptoms (i.e., high out-strength estimate) (Bos et al., 2017; Bringmann et al., 2015; Fried et al., 2016; Hakulinen et al., 2020). These findings are also in line with the current diagnostic approach (both ICD and DSM-V) which regards depressed mood and anhedonia as the core symptoms for a diagnosis of clinical depression (American Psychiatric Association, 2013; World Health Organization, 2004). 
Deterioration of sad or depressed mood was more likely to occur after deterioration of other symptoms; whereas improvement in sad or depressed mood was more likely to trigger improvements in other symptoms over time. Both improvement and deterioration of diminished interest was reactive to changes in other symptoms, and deterioration of diminished interest was also likely to induce deterioration in other symptoms over time. Previous longitudinal studies applying a directed network analysis have also shown that sadness and loss of interest were reactive to changes in other symptoms (Bos et al., 2017; Bringmann et al., 2015). However, in contrast to studies by Bos et al., 2017; Bringmann et al., 2015, absence of enjoyment did not play any central role in the symptom networks in our sample. Further studies are needed to investigate the (strength) centrality of sad or depressed mood and anhedonia in different samples and using different measures of depressive symptoms.

In line with our expectations, suicidal ideation also emerged as one of the most central symptoms in the networks of depressive symptoms. Suicidal ideation is among the strongest predictors of a diagnosis of severe depression which requires immediate attention and treatment (Malhi et al., 2014). We found that deterioration in suicidal ideation was most likely to induce deterioration in other symptoms over time. This accords with studies using directed network analyses (Bringmann et al., 2015) and direction of dependence methods (García-Velázquez et al., 2020) which suggest that suicidality is likely to reinforce other depressive symptoms.

It is not exactly known why and how antidepressant medication works (Harmer et al., 2009). Previous studies have shown that antidepressant medication may influence some symptoms differently than others (Hieronymus et al., 2016; Lisinski et al., 2020), but there have been no studies on whether antidepressant medication influences the strength of associations between depressive symptoms over time. We hypothesized that antidepressant 
medication might strengthen the positive effects of improvements between depressive symptoms and attenuate the negative effects of deterioration between depressive symptoms. We found no consistent differences in symptom networks between those taking versus not taking psychotropic medication. Two previous studies have also reported that the dynamic network structures of depressive symptoms (Bringmann et al., 2015) and mental states (Snippe et al., 2017) did not differ greatly in response to psychotherapy or antidepressant medication in small clinical trials. It should be noted, however, that the measure of medication use for depression or anxiety might have been too broad in this study, since no details on the type of medication, dosage, and length of use were available. It is therefore possible that no observed differences in the networks of depressive symptoms between 'treatment' and 'no treatment' groups reflect the imprecise definition of medication use. Our findings are based on a general population sample, and further research from clinical trials is needed to evaluate the role of antidepressant treatment in shaping the network structure of depressive symptoms.

\section{Limitations}

This study has some limitations which should be acknowledged. First, as with most longitudinal data, loss to follow-up was also inevitable in this study. The attrition analysis showed that participants reporting a higher number of depressive symptoms were more likely to drop out; therefore, the selective attrition might have biased our findings. Second, the measurements of depressive symptoms were conducted every two years. Depressive symptoms can vary greatly across long time periods (Eeden et al., 2019), and this may dilute some of the short-term temporal sequences between symptoms. However, our findings are similar to previous studies using intensive longitudinal data across days or weeks, suggesting that symptom-level analysis may be feasible with long-term follow-up data as well. Third, depressive symptoms were self-reported and thus involve the risk of reporting bias. This may 
be problematic for symptom-level analysis especially if the participants are not able to discriminate between different symptoms of depression in detail. Fourth, we did not have details regarding the medication use, such as dosage, length of use, or treatment adherence over time. Finally, our sample comprised people aged 50 or older which may limit the generalizability of our findings to younger cohorts.

\section{Conclusions}

In conclusion, symptom-level analysis over several years suggested that sad or depressed mood, diminished interest, and suicidal ideation may be the most central symptoms in the networks for changes in depressive symptoms over time. Psychotropic medication use did not modify these associations. 


\section{CRediT authorship contribution statement:}

Kateryna Savelieva: Conceptualization, Formal analysis, Writing - original draft, Writing - review and editing. Kaisla Komulainen: Conceptualization, Formal analysis, Writing - review and editing. Marko Elovainio: Conceptualization, Methodology, Writing review and editing, Funding acquisition. Markus Jokela: Conceptualization, Methodology, Supervision, Formal analysis, Writing - review and editing, Funding acquisition. All authors contributed to and have approved the final manuscript.

Funding: This work was supported by the Academy of Finland (grant number 311578 for MJ and 329224 for ME).

Acknowledgements: The SHARE data collection has been funded by the European Commission through FP5 (QLK6-CT-2001-00360), FP6 (SHARE-I3: RII-CT-2006-062193, COMPARE: CIT5-CT-2005-028857, SHARELIFE: CIT4-CT-2006-028812), FP7 (SHAREPREP: GA N²11909, SHARE-LEAP: GA N²27822, SHARE M4: GA N²61982) and Horizon 2020 (SHARE-DEV3: GA N676536, SERISS: GA N654221) and by DG Employment, Social Affairs \& Inclusion. Additional funding from the German Ministry of Education and Research, the Max Planck Society for the Advancement of Science, the U.S. National Institute on Aging (U01_AG09740-13S2, P01_AG005842, P01_AG08291, P30_AG12815, R21_AG025169, Y1-AG-4553-01, IAG_BSR06-11, OGHA_04-064, HHSN271201300071C) and from various national funding sources is gratefully acknowledged (see www.share-project.org).

Data availability statement: The data that support the findings of this study are distributed by SHARE-ERIC to registered users through the SHARE Research Data Center 
(https://releases.sharedataportal.eu/users/login). This paper uses data from SHARE Waves 1, 2, 4, 5, 6 and 7 (DOIs: 10.6103/SHARE.w1.700, 10.6103/SHARE.w2.700, $\underline{\text { 10.6103/SHARE.w4.700, 10.6103/SHARE.w5.700, 10.6103/SHARE.w6.700, }}, \underline{10.6103 / \mathrm{SHA}}$ RE.w7.700).

Ethical standard: The authors assert that all procedures contributing to this work comply with the ethical standards of the relevant national and institutional committees on human experimentation and with the Helsinki Declaration of 1975, as revised in 2008. 


\section{References}

American Psychiatric Association, 2013. Diagnostic and Statistical Manual of Mental

Disorders. American Psychiatric Association.

https://doi.org/10.1176/appi.books.9780890425596

Bergmann, M., Kneip, T., De Luca, G., Scherpenzeel, A., 2019. Survey participation in the Survey of Health, Ageing and Retirement in Europe (SHARE), Wave 1-7. Based on Release 7.0.0., SHARE Working Paper Series (41-2019). Munich.

Borsboom, D., 2017. A network theory of mental disorders. World Psychiatry 16, 5-13. https://doi.org/10.1002/wps.20375

Börsch-Supan, A., 2019a. Survey of Health, Ageing and Retirement in Europe (SHARE) Wave 1. Release version: 7.0.0. SHARE-ERIC. Data set. DOI: 10.6103/SHARE.w1.700.

Börsch-Supan, A., 2019b. Survey of Health, Ageing and Retirement in Europe (SHARE) Wave 2. Release version: 7.0.0. SHARE-ERIC. Data set. DOI: 10.6103/SHARE.w2.700.

Börsch-Supan, A., 2019c. Survey of Health, Ageing and Retirement in Europe (SHARE) Wave 4. Release version: 7.0.0. SHARE-ERIC. Data set. DOI: 10.6103/SHARE.w4.700.

Börsch-Supan, A., 2019d. Survey of Health, Ageing and Retirement in Europe (SHARE) Wave 5. Release version: 7.0.0. SHARE-ERIC. Data set. DOI: 10.6103/SHARE.w5.700.

Börsch-Supan, A., 2019e. Survey of Health, Ageing and Retirement in Europe (SHARE) Wave 6. Release version: 7.0.0. SHARE-ERIC. Data set. DOI: 10.6103/SHARE.w6.700.

Börsch-Supan, A., 2019f. Survey of Health, Ageing and Retirement in Europe (SHARE) 
Wave 7. Release version: 7.0.0. SHARE-ERIC. Data set. DOI: 10.6103/SHARE.w7.700.

Börsch-Supan, A., Brandt, M., Hunkler, C., Kneip, T., Korbmacher, J., Malter, F., Schaan, B., Stuck, S., Zuber, S., 2013. Data resource profile: The Survey of Health, Ageing and Retirement in Europe (SHARE). Int. J. Epidemiol. 42, 992-1001. https://doi.org/10.1093/ije/dyt088

Bos, F.M., Snippe, E., de Vos, S., Hartmann, J.A., Simons, C.J.P., van der Krieke, L., de Jonge, P., Wichers, M., 2017. Can we jump from cross-sectional to dynamic interpretations of networks? Implications for the network perspective in psychiatry. Psychother. Psychosom. 86, 175-177. https://doi.org/10.1159/000453583

Bringmann, L.F., Lemmens, L.H.J.M., Huibers, M.J.H., Borsboom, D., Tuerlinckx, F., 2015. Revealing the dynamic network structure of the Beck Depression Inventory-II. Psychol. Med. 45, 747-757. https://doi.org/10.1017/S0033291714001809

Castro-Costa, E., Dewey, M., Stewart, R., Banerjee, S., Huppert, F., Mendonca-Lima, C., Bula, C., Reisches, F., Wancata, J., Ritchie, K., Tsolaki, M., Mateos, R., Prince, M., 2008. Ascertaining late-life depressive symptoms in Europe: An evaluation of the survey version of the EURO-D scale in 10 nations. The SHARE project. Int. J. Methods Psychiatr. Res. 17, 12-29. https://doi.org/10.1002/mpr.236

Cramer, A.O.J., Waldorp, L.J., van der Maas, H.L.J., Borsboom, D., 2010. Comorbidity: A network perspective. Behav. Brain Sci. 33, 137-150. https://doi.org/10.1017/S0140525X09991567

Eeden, W.A., Hemert, A.M., Carlier, I.V.E., Penninx, B.W., Giltay, E.J., 2019. Severity, course trajectory, and within-person variability of individual symptoms in patients with major depressive disorder. Acta Psychiatr. Scand. 139, 194-205. https://doi.org/10.1111/acps.12987 
Fried, E.I., 2017. The 52 symptoms of major depression: Lack of content overlap among seven common depression scales. J. Affect. Disord. 208, 191-197. https://doi.org/10.1016/j.jad.2016.10.019

Fried, E.I., Epskamp, S., Nesse, R.M., Tuerlinckx, F., Borsboom, D., 2016. What are "good" depression symptoms? Comparing the centrality of DSM and non-DSM symptoms of depression in a network analysis. J. Affect. Disord. 189, 314-320. https://doi.org/10.1016/j.jad.2015.09.005

Fried, E.I., Nesse, R.M., 2015. Depression sum-scores don't add up: Why analyzing specific depression symptoms is essential. BMC Med. 13, 72. https://doi.org/10.1186/s12916015-0325-4

Fried, E.I., van Borkulo, C.D., Cramer, A.O.J., Boschloo, L., Schoevers, R.A., Borsboom, D., 2017. Mental disorders as networks of problems: A review of recent insights. Soc. Psychiatry Psychiatr. Epidemiol. 52, 1-10. https://doi.org/10.1007/s00127-016-1319-z

García-Velázquez, R., Jokela, M., Rosenström, T.H., 2020. Direction of dependence between specific symptoms of depression: A Non-Gaussian approach. Clin. Psychol. Sci. 8, 240 251. https://doi.org/10.1177/2167702619875410

Groen, R.N., Snippe, E., Bringmann, L.F., Simons, C.J.P., Hartmann, J.A., Bos, E.H., Wichers, M., 2019. Capturing the risk of persisting depressive symptoms: A dynamic network investigation of patients' daily symptom experiences. Psychiatry Res. 271, 640-648. https://doi.org/10.1016/j.psychres.2018.12.054

Hakulinen, C., Fried, E.I., Pulkki-Råback, L., Virtanen, M., Suvisaari, J., Elovainio, M., 2020. Network structure of depression symptomology in participants with and without depressive disorder: The population-based Health 2000-2011 study. Soc. Psychiatry Psychiatr. Epidemiol. https://doi.org/10.1007/s00127-020-01843-7

Harmer, C.J., Goodwin, G.M., Cowen, P.J., 2009. Why do antidepressants take so long to 
work? A cognitive neuropsychological model of antidepressant drug action. Br. J. Psychiatry 195, 102-108. https://doi.org/10.1192/bjp.bp.108.051193

Hieronymus, F., Emilsson, J.F., Nilsson, S., Eriksson, E., 2016. Consistent superiority of selective serotonin reuptake inhibitors over placebo in reducing depressed mood in patients with major depression. Mol. Psychiatry 21, 523-530. https://doi.org/10.1038/mp.2015.53

Klippel, A., Viechtbauer, W., Reininghaus, U., Wigman, J., van Borkulo, C., Myin-Germeys, I., Wichers, M., 2018. The cascade of stress: A network approach to explore differential dynamics in populations varying in risk for psychosis. Schizophr. Bull. 44, 328-337. https://doi.org/10.1093/schbul/sbx037

Lewer, D., O’Reilly, C., Mojtabai, R., Evans-Lacko, S., 2015. Antidepressant use in 27 European countries: Associations with sociodemographic, cultural and economic factors. Br. J. Psychiatry 207, 221-226. https://doi.org/10.1192/bjp.bp.114.156786

Lisinski, A., Hieronymus, F., Näslund, J., Nilsson, S., Eriksson, E., 2020. Item-based analysis of the effects of duloxetine in depression: A patient-level post hoc study.

Neuropsychopharmacology 45, 553-560. https://doi.org/10.1038/s41386-019-0523-4

Malhi, G.S., Coulston, C.M., Fritz, K., Lampe, L., Bargh, D.M., Ablett, M., Lyndon, B., Sapsford, R., Theodoros, M., Woolfall, D., van der Zypp, A., Hopwood, M., Mitchell, A.J., 2014. Unlocking the diagnosis of depression in primary care: Which key symptoms are GPs using to determine diagnosis and severity? Aust. New Zeal. J. Psychiatry 48, 542-547. https://doi.org/10.1177/0004867413513342

Prince, M.J., Beekman, A.T.F., Deeg, D.J.H., Fuhrer, R., Kivela, S.-L., Lawlor, B.A., Lobo, A., Magnusson, H., Meller, I., Van Oyen, H., Reischies, F., Roelands, M., Skoog, I., Turrina, C., Copeland, J.R.M., 1999a. Depression symptoms in late life assessed using the EURO-D scale. Br. J. Psychiatry 174, 339-345. 
https://doi.org/10.1192/bjp.174.4.339

Prince, M.J., Reischies, F., Beekman, A.T.F., Fuhrer, R., Jonker, C., Kivela, S.-L., Lawlor, B.A., Lobo, A., Magnusson, H., Fichter, M., Van Oyen, H., Roelands, M., Skoog, I., Turrina, C., Copeland, J.R.M., 1999b. Development of the EURO-D scale - a European Union initiative to compare symptoms of depression in 14 European centres. Br. J. Psychiatry 174, 330-338. https://doi.org/10.1192/bjp.174.4.330

RStudio Team, 2015. RStudio: Integrated Development for R. RStudio, Inc., Boston, MA URL http://www.rstudio.com/.

Snippe, E., Viechtbauer, W., Geschwind, N., Klippel, A., de Jonge, P., Wichers, M., 2017. The impact of treatments for depression on the dynamic network structure of mental states: Two randomized controlled trials. Sci. Rep. 7, 46523. https://doi.org/10.1038/srep46523

StataCorp, 2017. Stata Statistical Software: Release 15.

World Health Organization, 2004. ICD-10: International statistical classification of diseases and related health problems: Tenth revision, 2nd ed., World Health Organization. 


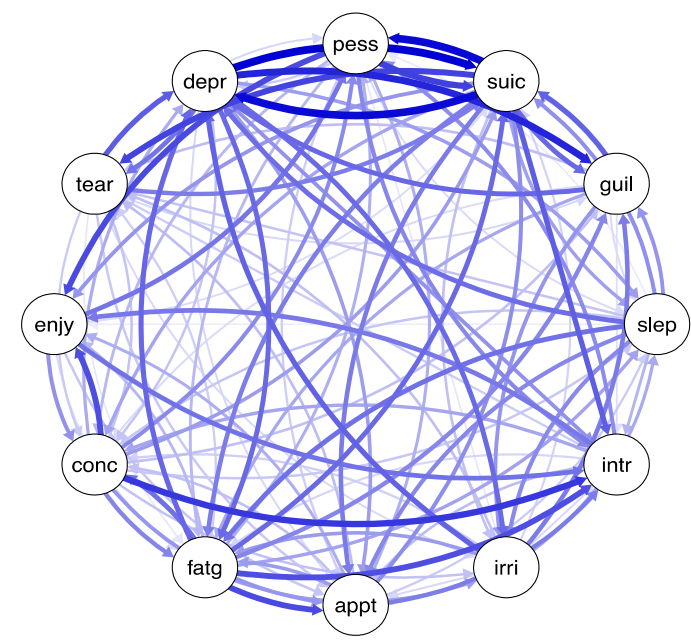

(A)

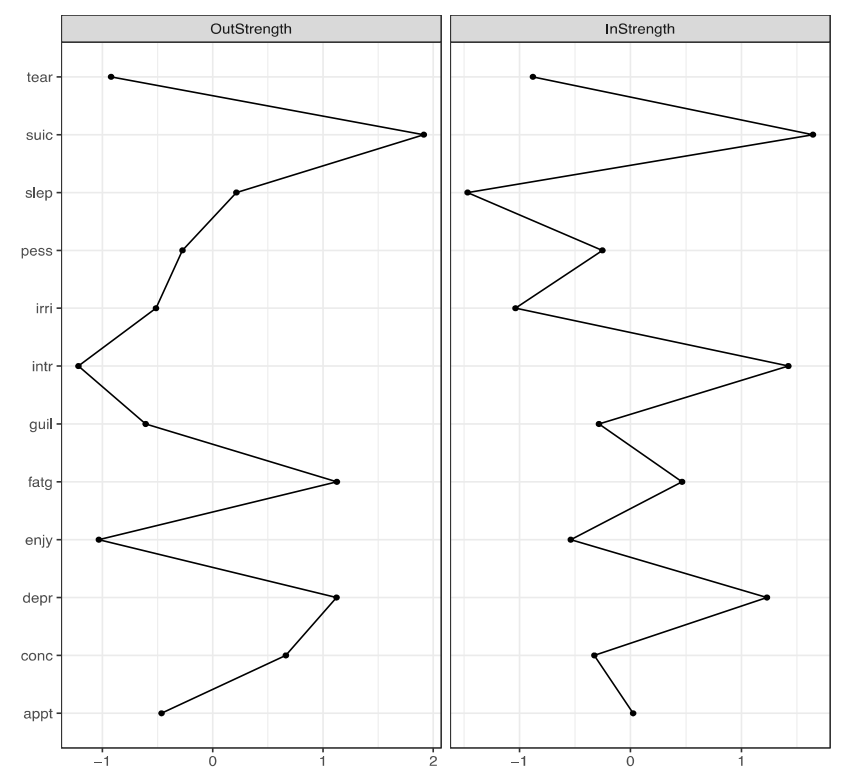

(B)

Figure 1. (A) Dynamic network of depressive symptoms and (B) centrality indices for the network of depressive symptoms based on the time-lag model in the total sample $(n=146,470)$. Positive associations are shown in blue; line (edge) thickness indicates weight. Note. Depr: sad or depressed last month; pess: pessimism (no hopes for the future); suic: felt would rather be dead; guil: feels guilty; slep: trouble sleeping; intr: less interest in things; irri: irritability; appt: appetite; fatg: fatigue; conc: difficulty in concentrating on entertainment or reading; enjy: enjoyment (fails to mention any enjoyable activity); tear: tearfulness. 


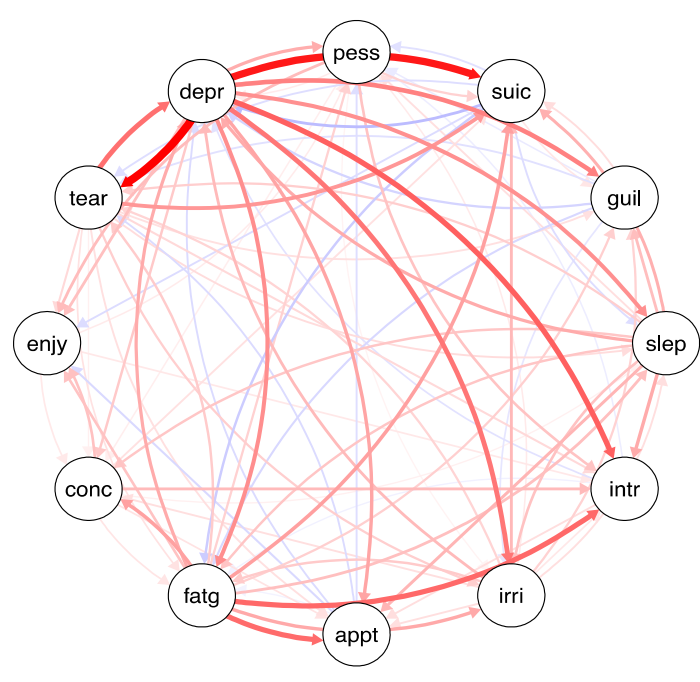

(A)

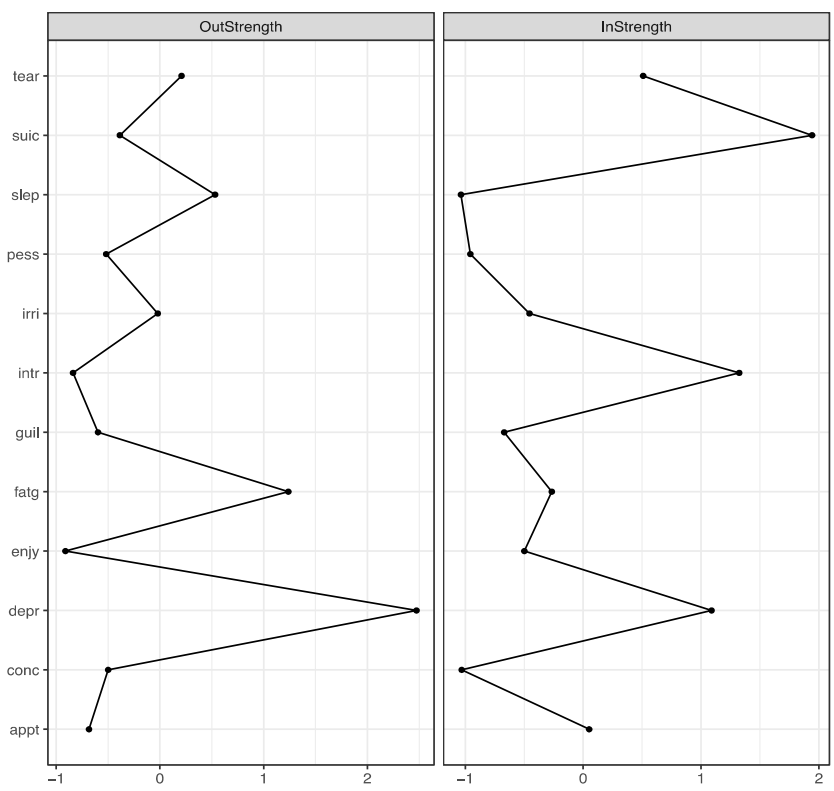

(B)

Figure 2. (A) Dynamic network of subsequent improvement in depressive symptoms and (B) centrality indices for this network based on the change model in the total sample. Negative associations in red indicate improvement of symptoms, and positive associations in blue deterioration of symptoms; line (edge) thickness indicates weight.

Note. Depr: sad or depressed last month; pess: pessimism (no hopes for the future); suic: felt would rather be dead; guil: feels guilty; slep: trouble sleeping; intr: less interest in things; irri: irritability; appt: appetite; fatg: fatigue; conc: difficulty in concentrating on entertainment or reading; enjy: enjoyment (fails to mention any enjoyable activity); tear: tearfulness. 


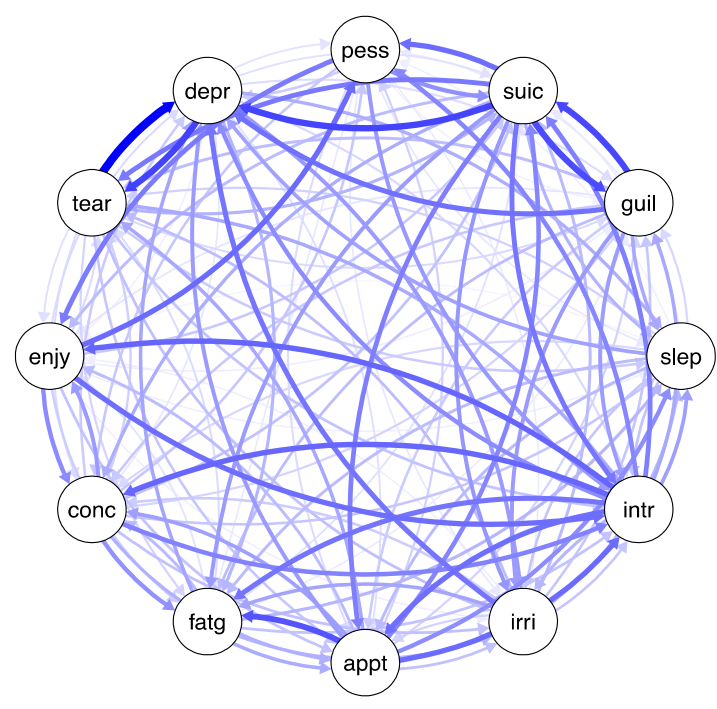

(A)

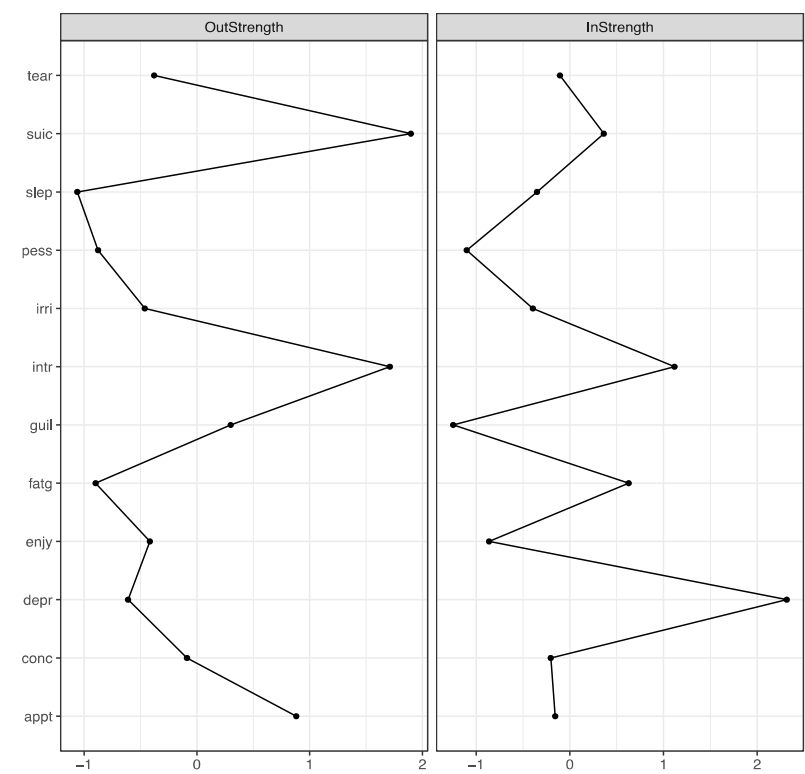

(B)

Figure 3. (A) Dynamic network of subsequent deterioration of depressive symptoms and (B) centrality indices for this network based on the change model in the total sample. Positive associations in blue indicate deterioration of symptoms, and negative associations in red improvement of symptoms; line (edge) thickness indicates weight.

Note. Depr: sad or depressed last month; pess: pessimism (no hopes for the future); suic: felt would rather be dead; guil: feels guilty; slep: trouble sleeping; intr: less interest in things; irri: irritability; appt: appetite; fatg: fatigue; conc: difficulty in concentrating on entertainment or reading; enjy: enjoyment (fails to mention any enjoyable activity); tear: tearfulness. 
(A) Improvement

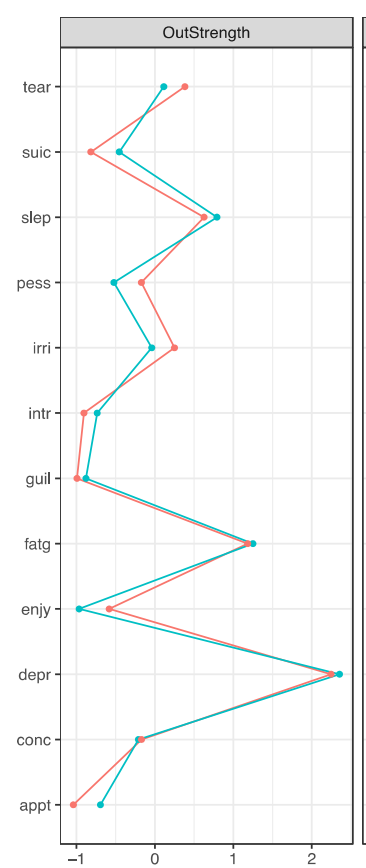

(B) Deterioration

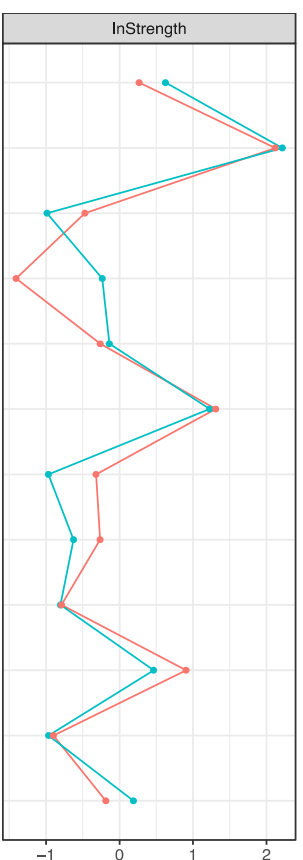

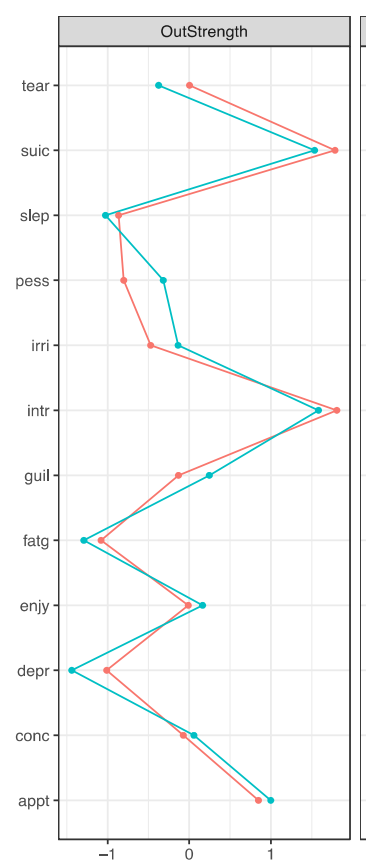

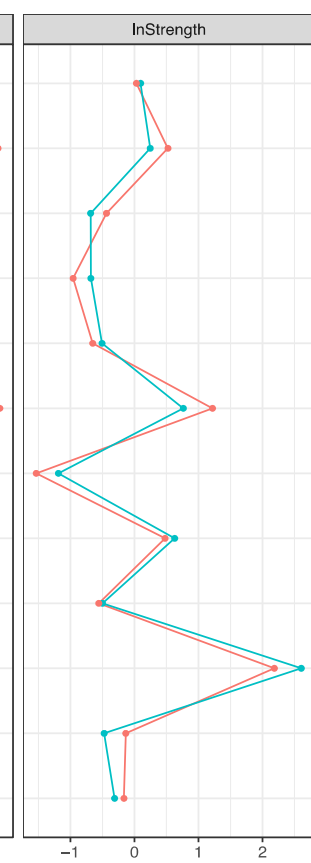

уур

_treatment_group

$\rightarrow$ Treatment_group

Figure 4. Centrality indices for the networks of depressive symptoms in 'treatment group' and 'no treatment group' based on the change model ((A) improvement of symptoms over time, (B) deterioration of symptoms over time). 